\title{
ON THE LINEAR COMBINANTS OF A BINARY PENCIL
}

\author{
ABDELMALEK ABDESSELAM \\ Kerchof Hall Department of Mathematics, University of Virginia, PO Box 400137, \\ Charlottesville, VA 22904-4137, USA \\ e-mail:malek@virginia.edu \\ and JAYDEEP CHIPALKATTI \\ Machray Hall, Department of Mathematics, University of Manitoba, Winnipeg MB R3T 2N2, Canada \\ e-mail: chipalka@cc.umanitoba.ca
}

(Received 16 June 2008; revised 24 October 2008; accepted 17 December 2008)

\begin{abstract}
Let $A, B$ denote binary forms of order $d$, and let $\mathcal{C}_{2 r-1}=(A, B)_{2 r-1}$ be the sequence of their linear combinants for $1 \leq r \leq\left\lfloor\frac{d+1}{2}\right\rfloor$. It is known that $\mathcal{C}_{1}, \mathcal{C}_{3}$ together determine the pencil $\{A+\lambda B\}_{\lambda \in \mathbf{P}^{1}}$ and hence indirectly the higher combinants $\mathcal{C}_{2 r-1}$. In this paper we exhibit explicit formulae for all $r \geq 3$, which allow us to recover $\mathcal{C}_{2 r-1}$ from the knowledge of $\mathcal{C}_{1}$ and $\mathcal{C}_{3}$. The calculations make use of the symbolic method in classical invariant theory, as well as the quantum theory of angular momentum. Our theorem pertains to the plethysm representation $\wedge^{2} S_{d}$ for the group $S L_{2}$. We give an example for the group $S L_{3}$ to show that such a result may hold for other categories of representations.
\end{abstract}

2000 Mathematics Subject Classification. 13A50, 22E70.

1. Introduction. This paper is a thematic sequel to [3] and [5]. The problem solved here was originally posed in [5] (of which a précis is given below). All of the unexplained notation and terminology used in this paper may be found in [3]. The reader is referred to $[6,7,11,14]$ for foundational material in classical invariant theory and the symbolic method. The basics of the representation theory of $S L_{2}$ may be found in [8, Lecture 11] and [17, Chapter 4].

1.1. Transvectants. The base field $\mathbb{k}$ will be of characteristic zero. Let $S_{d}$ denote the $(d+1)$-dimensional irreducible representation of the group $S L_{2}=S L(2, \mathbb{k})$. We identify $S_{d}$ with the space of (homogeneous) binary $d$-ics in the variables $\mathbf{x}=\left(x_{1}, x_{2}\right)$.

Given integers $m, n \geq 0$ and $0 \leq q \leq \min (m, n)$, there is an $S L_{2}$-equivariant split surjection (see [3, Section 1.5])

$$
\pi_{q}: S_{m} \otimes S_{n} \longrightarrow S_{m+n-2 q}
$$

Given binary forms $F \in S_{m}$ and $G \in S_{n}$, the image $\pi_{q}(F \otimes G)$ is classically referred to as the $q$ th transvectant of $F$ and $G$, denoted by $(F, G)_{q}$. We have an explicit formula

$$
(F, G)_{q}=\frac{(m-q) !(n-q) !}{m ! n !} \sum_{i=0}^{q}(-1)^{i}\left(\begin{array}{l}
q \\
i
\end{array}\right) \frac{\partial^{q} F}{\partial x_{1}^{q-i} \partial x_{2}^{i}} \frac{\partial^{q} G}{\partial x_{1}^{i} \partial x_{2}^{q-i}}
$$


however, it is seldom directly useful. For later use, let

$$
\imath_{q}: S_{m+n-2 q} \longrightarrow S_{m} \otimes S_{n}
$$

denote the canonical inclusion, so that $\pi_{q} \circ l_{q}$ is the identity map on $S_{m+n-2 q}$.

1.2. Combinants. Now let $A, B \in S_{d}$ denote two linearly independent forms. There is an isomorphism of $S L_{2}$-representations

$$
\wedge^{2} S_{d}=\bigoplus_{r=1}^{\left\lfloor\frac{d+1}{2}\right\rfloor} S_{2 d-4 r+2},
$$

with projection morphisms $p_{r}: \wedge^{2} S_{d} \longrightarrow S_{2 d-4 r+2}$. The image $p_{r}(A \wedge B)$ equals the transvectant $(A, B)_{2 r-1}$. For any scalar $\alpha, \beta, \gamma, \delta$, we have an invariance property

$$
(\alpha A+\beta B, \gamma A+\delta B)_{2 r-1}=(\alpha \delta-\beta \gamma)(A, B)_{2 r-1} .
$$

Hence, up to a scalar, the forms $\mathcal{C}_{2 r-1}=(A, B)_{2 r-1}$ depend only on the subspace $\Pi_{A, B}=\operatorname{Span}\{A, B\}$. In classical terminology (see [11, Section 250]), $\left\{\mathcal{C}_{2 r-1}\right\}$ are linear combinants of the pencil $\{A+\lambda B\}_{\lambda \in \mathbf{P}^{1}}$.

Decomposition (3) implies that the pencil is completely determined by the sequence of forms

$$
\mathcal{C}_{1}, \mathcal{C}_{3}, \ldots, \mathcal{C}_{\left\lfloor\frac{d+1}{2}\right\rfloor}
$$

This claim can be sharpened. Recall that an arbitrary form $F \in S_{d}$ belongs to $\Pi_{A, B}$, if and only if the Wronskian

$$
\mathbf{W}=\left|\begin{array}{lll}
A_{x_{1}^{2}} & A_{x_{1} x_{2}} & A_{x_{2}^{2}} \\
B_{x_{1}^{2}} & B_{x_{1} x_{2}} & B_{x_{2}^{2}} \\
F_{x_{1}^{2}} & F_{x_{1} x_{2}} & F_{x_{2}^{2}}
\end{array}\right|
$$

is identically zero. A symbolic calculation shows that (see [5, Proposition 3.2])

$$
\frac{1}{\left(d^{2}-d\right)^{3}} \mathbf{W}=\left(\mathcal{C}_{1}, F\right)_{2}-\frac{d-2}{4 d-6} F \mathcal{C}_{3}
$$

Hence the condition $F \in \Pi_{A, B}$ is expressible only in terms of $\mathcal{C}_{1}, \mathcal{C}_{3}$ and $F$. It follows that $\mathcal{C}_{1}, \mathcal{C}_{3}$ determine $\Pi_{A, B}$, and hence they indirectly determine all the subsequent combinants $\mathcal{C}_{5}, \mathcal{C}_{7}, \mathcal{C}_{9}$, etc. It is natural to enquire whether there exists a concrete formula for $\mathcal{C}_{2 r-1}$ in terms of $\mathcal{C}_{1}$ and $\mathcal{C}_{3}$. This problem was solved in [5, Section 5] for $\mathcal{C}_{5}$ and $\mathcal{C}_{7}$, using some ad hoc calculations; here we will give an inductive solution which applies to all $r \geq 3$.

EXAmple 1.1. Assume $d=7$. We have an identity

$$
\mathcal{C}_{1} \mathcal{C}_{5}=-\frac{21}{2}\left(\mathcal{C}_{1}, \mathcal{C}_{1}\right)_{4}+\frac{84}{11}\left(\mathcal{C}_{1}, \mathcal{C}_{3}\right)_{2}+\frac{735}{484} \mathcal{C}_{3}^{2}
$$


which expresses $\mathcal{C}_{5}$ in terms of $\mathcal{C}_{1}, \mathcal{C}_{3}$. Similarly, the identity

$$
\begin{aligned}
\mathcal{C}_{1} \mathcal{C}_{7}= & -28\left(\mathcal{C}_{1}, \mathcal{C}_{1}\right)_{6}-\frac{210}{11}\left(\mathcal{C}_{1}, \mathcal{C}_{3}\right)_{4}+8\left(\mathcal{C}_{1}, \mathcal{C}_{5}\right)_{2} \\
& +\frac{1960}{121}\left(\mathcal{C}_{3}, \mathcal{C}_{3}\right)_{2}+\frac{35}{11} \mathcal{C}_{3} \mathcal{C}_{5}
\end{aligned}
$$

indirectly expresses $\mathcal{C}_{7}$ in terms of $\mathcal{C}_{1}, \mathcal{C}_{3}$. We will show that such formulae always exist for all $d$ and $3 \leq r \leq\left\lfloor\frac{d+1}{2}\right\rfloor$.

After completing our results, we discovered that a few such calculations had been done by Shenton [15, p. 257ff.].

2. Quadratic syzygies. Define a (quadratic) syzygy of weight $2 r$ to be an identity

$$
\sum \alpha_{i, j}\left(\mathcal{C}_{2 i-1}, \mathcal{C}_{2 j-1}\right)_{2(r-i-j+1)}=0 \quad\left(\alpha_{i, j} \in \mathbb{k}\right)
$$

assumed to hold for all $d$-ics $A, B$. The sum is quantified over all pairs $(i, j)$ such that

$$
1 \leq i \leq j \leq r, \quad i+j \leq r+1 .
$$

For instance, (4) and (5) are syzygies of weight 6 and 8 respectively. Notice that the only term in (6) involving $\mathcal{C}_{2 r-1}$ corresponds to $(i, j)=(1, r)$. Now our main result is the following:

THEOREM 2.1. For every $3 \leq r \leq\left\lfloor\frac{d+1}{2}\right\rfloor$, there exists a quadratic syzygy of weight $2 r$ such that $\alpha_{1, r} \neq 0$.

We will, in fact, produce an explicit formula for the $\alpha_{i, j}$. Given this, one can rewrite (6) as

$$
\mathcal{C}_{2 r-1}=-\frac{1}{\mathcal{C}_{1}} \sum \frac{\alpha_{i, j}}{\alpha_{1, r}}\left(\mathcal{C}_{2 i-1}, \mathcal{C}_{2 j-1}\right)_{2(r-i-j+1)}=0
$$

which recovers $\mathcal{C}_{2 r-1}$ from $\mathcal{C}_{1}, \ldots, \mathcal{C}_{2 r-3}$. Notice that $\mathcal{C}_{1}$ is (up to a scalar) the Jacobian of $A, B$; in particular it is non-zero if $\{A, B\}$ are linearly independent.

By a classical theorem of Gordan, the algebra of all combinants of a pencil is finitely generated. However, a specific set of generators is known in only a few cases (see $[10,12,13,18])$. Our main theorem is not directly comparable to these results, since we allow not only polynomial but also rational transvectant expressions in the combinants.

2.1. An outline of the proof. The following proposition (proved in [5, Section 5]) reinterprets a syzygy as an $S L_{2}$-equivariant morphism.

Proposition 2.2. The vector space of syzygies of weight $2 r$ is isomorphic to $\operatorname{Hom}_{S L_{2}}\left(S_{4(d-r)}, \wedge^{4} S_{d}\right)$.

In Section 2.5 we will construct a specific morphism

$$
\zeta: S_{4(d-r)} \longrightarrow \wedge^{4} S_{d}
$$


and then calculate the corresponding syzygy coefficients. This calculation will be done twice: first by classical symbolic methods and second by recasting the coefficient as a 9-j symbol in the sense of the quantum theory of angular momentum.

It would be of interest to know whether an analogue of Theorem 2.1 holds for other categories of representations. In Section 4 we give such an example for the group $\mathrm{SL}_{3}$.

2.2. The Plücker imbedding. We informally sketch the idea behind Proposition 2.2. Consider the Plücker imbedding

$$
G\left(2, S_{d}\right) \hookrightarrow \mathbf{P}\left(\wedge^{2} S_{d}\right),
$$

with image $X$ and ideal sheaf $\mathcal{I}_{X}$. The short exact sequence of $S L_{2}$-representations

$$
0 \rightarrow H^{0}\left(\mathcal{I}_{X}(2)\right) \rightarrow H^{0}\left(\mathcal{O}_{\mathbf{P}}(2)\right) \rightarrow H^{0}\left(\mathcal{O}_{X}(2)\right) \rightarrow 0
$$

can be naturally identified with

$$
0 \rightarrow \wedge^{4} S_{d} \stackrel{l}{\rightarrow} S_{2}\left(\wedge^{2} S_{d}\right) \stackrel{q}{\rightarrow} \mathbb{S}_{(2,2)}\left(S_{d}\right) \rightarrow 0
$$

Here $\mathbb{S}_{(2,2)}$ denotes the Schur functor associated to the partition $(2,2)$ (see $[8$, Lecture 6]). The coefficients of each $\mathcal{C}_{2 i-1}$ can be seen as $\mathbb{k}$-linear combinations of homogeneous coordinates on $\mathbf{P}\left(\wedge^{2} S_{d}\right)$ (see [2, Example 5.3]). Now consider an arbitrary expression of the form

$$
\mathcal{E}=\sum \alpha_{i, j}\left(\mathcal{C}_{2 i-1}, \mathcal{C}_{2 j-1}\right)_{2(r-i-j+1)} \quad\left(\alpha_{i, j} \in \mathbb{k}\right)
$$

It may be seen as defining a morphism

$$
S_{4(d-r)} \stackrel{\phi_{\mathcal{E}}}{\longrightarrow} H^{0}\left(\mathcal{O}_{\mathbf{P}}(2)\right), \quad F \longrightarrow(F, \mathcal{E})_{4(d-r)}
$$

Indeed, note that the transvectant $(F, \mathcal{E})_{4(d-r)}$ does not involve the $\mathbf{x}$-variables. For a fixed $F$, it is a homogeneous quadratic expression in the Plücker coordinates and as such gives a global section of $\mathcal{O}_{\mathbf{P}}(2)$. Now $\mathcal{E}$ is a syzygy exactly when this section is identically zero when restricted to $X$, i.e. iff $q \circ \phi_{\mathcal{E}}=0$. This is equivalent to the condition that $\phi_{\mathcal{E}}$ factor through ker $q$. Conversely, a non-zero map $S_{4(d-r)} \stackrel{\phi}{\longrightarrow} \wedge{ }^{4} S_{d}$ defines an irreducible sub-representation of $H^{0}\left(\mathcal{I}_{X}(2)\right)$, which translates into a quadratic syzygy $\mathcal{E}_{\phi}$.

2.3. Syzygy coefficients. This interpretation allows to read off the individual coefficients in a syzygy. Let $\mathcal{E}=0$ denote a quadratic syzygy of weight $2 r$, and fix a pair of integers $(i, j)$ satisfying

$$
1 \leq i, j \leq r, \quad i+j \leq r+1 .
$$


(Notice that we have not imposed the condition $i \leq j$.) Consider the sequence of morphisms

$$
\begin{gathered}
S_{4(d-r)} \stackrel{\phi_{\mathcal{E}}}{\longrightarrow} \wedge^{4} S_{d} \stackrel{l}{\longrightarrow} S_{2}\left(\wedge^{2} S_{d}\right) \stackrel{\beta_{1}}{\longrightarrow} \wedge^{2} S_{d} \otimes \wedge^{2} S_{d} \\
\stackrel{\beta_{2}}{\longrightarrow} S_{2 d-4 i+2} \otimes S_{2 d-4 j+2} \stackrel{\beta_{3}}{\longrightarrow} S_{4(d-r)} .
\end{gathered}
$$

Here $\beta_{1}$ is the natural inclusion map $v \cdot w \longrightarrow \frac{1}{2}(v \otimes w+w \otimes v) ; \beta_{2}$ is the tensor product of projections $p_{2 i-1} \otimes p_{2 j-1}$; and $\beta_{3}$ is the transvectant map $\pi_{2(r-i-j+1)}$. By Schur's lemma, the composite endomorphism

$$
\beta_{3} \circ \beta_{2} \circ \beta_{1} \circ \imath \circ \phi_{\mathcal{E}}: S_{4(d-r)} \longrightarrow S_{4(d-r)}
$$

must be the multiplication by a constant, say $\theta_{i, j}$. Then, up to a global constant,

$$
\mathcal{E}=\sum \theta_{i, j}\left(\mathcal{C}_{2 i-1}, \mathcal{C}_{2 j-1}\right)_{2(r-i-j+1)}
$$

2.4. Symbolic description of the $\beta_{i}$. In this section we will describe $\beta_{i}$ using the classical symbolic calculus. Our notation follows [3] and [11]; in particular, the boldface letters $\mathbf{x}, \mathbf{y}, \mathbf{t}$, etc. stand for pairs of binary variables, and

$$
(\mathbf{x y})=x_{1} y_{2}-y_{1} x_{2}, \quad f_{\mathbf{t}}=f_{1} t_{1}+f_{2} t_{2}, \text { etc. }
$$

Cayley's Omega operator is defined to be

$$
\Omega_{\mathbf{x y}}=\frac{\partial^{2}}{\partial x_{1} \partial y_{2}}-\frac{\partial^{2}}{\partial y_{1} \partial x_{2}} .
$$

Let

$$
\mathrm{h}(m, n ; q)=\frac{(m+n-2 q+1) !}{(m+n-q+1) ! q !} .
$$

The rationale for introducing this factor is explained in [3, Section 1.6].

We will realise $S_{2}\left(\wedge^{2} S_{d}\right)$ as the space of quadri-homogeneous forms $Q(\mathbf{x}, \mathbf{y}, \mathbf{z}, \mathbf{w})$ of order $d$ in each variable, satisfying the conditions

$$
Q(\mathbf{x}, \mathbf{y}, \mathbf{z}, \mathbf{w})=-Q(\mathbf{y}, \mathbf{x}, \mathbf{z}, \mathbf{w})=-Q(\mathbf{x}, \mathbf{y}, \mathbf{w}, \mathbf{z})=Q(\mathbf{z}, \mathbf{w}, \mathbf{x}, \mathbf{y}) .
$$

Inside this space, the image of $l$ is identified with the set of alternating forms, i.e. those $Q$ for which

$$
Q(\mathbf{x}, \mathbf{y}, \mathbf{z}, \mathbf{w})=\operatorname{sign}(\sigma) Q\left(\mathbf{x}^{\sigma}, \mathbf{y}^{\sigma}, \mathbf{z}^{\sigma}, \mathbf{w}^{\sigma}\right),
$$

for every permutation $\sigma$ of the four letters.

Now realise $S_{2 d-4 i+2} \otimes S_{2 d-4 j+2}$ as the space of bi-homogeneous forms of respective orders $(2 d-4 i+2,2 d-4 j+2)$ in $\mathbf{u}, \mathbf{v}$. Then $\beta_{2} \circ \beta_{1}$ maps $Q$ to

$$
\mathrm{h}(d, d ; 2 i-1) \mathrm{h}(d, d ; 2 j-1)\left[\Omega_{\mathbf{x y}}^{2 i-1} \Omega_{\mathbf{z w}}^{2 j-1} Q\right]
$$


followed by the substitutions $\{\mathbf{x}, \mathbf{y} \rightarrow \mathbf{u}\},\{\mathbf{z}, \mathbf{w} \rightarrow \mathbf{v}\}$. Notice that, given the two pairs of operations

$$
\Omega_{\mathbf{x y}},\{\mathbf{x}, \mathbf{y} \rightarrow \mathbf{u}\}, \quad \Omega_{\mathbf{z w}},\{\mathbf{z}, \mathbf{w} \rightarrow \mathbf{v}\}
$$

any operation from the first pair commutes from any operation from the second. Finally realise $S_{4(d-r)}$ as order $4(d-r)$ forms in $\mathbf{t}$; then $\beta_{3}$ maps $R(\mathbf{u}, \mathbf{v})$ to

$$
\mathrm{h}(2 d-4 i+2,2 d-4 j+2 ; 2 r-2 i-2 j+2)\left[\Omega_{\mathbf{u v}}^{2 r-2 i-2 j+2} R(\mathbf{u}, \mathbf{v})\right]
$$

followed by the substitutions $\{\mathbf{u}, \mathbf{v} \rightarrow \mathbf{t}\}$.

2.5. The definition of $\zeta$. Now define $\zeta: S_{4(d-r)} \longrightarrow S_{2}\left(\wedge^{2} S_{d}\right)$ to be the morphism which sends $f_{\mathbf{t}}^{4(d-r)}$ to the form

$$
\begin{aligned}
\mathcal{F}= & (\mathbf{x} \mathbf{y})(\mathbf{z ~ w})^{2 r-1} f_{\mathbf{x}}^{d-1} f_{\mathbf{y}}^{d-1} f_{\mathbf{z}}^{d-2 r+1} f_{\mathbf{w}}^{d-2 r+1} \\
& -(\mathbf{x ~ z})(\mathbf{y} \mathbf{w})^{2 r-1} f_{\mathbf{x}}^{d-1} f_{\mathbf{z}}^{d-1} f_{\mathbf{y}}^{d-2 r+1} f_{\mathbf{w}}^{d-2 r+1} \\
& +(\mathbf{x ~ w})(\mathbf{y} \mathbf{z})^{2 r-1} f_{\mathbf{x}}^{d-1} f_{\mathbf{w}}^{d-1} f_{\mathbf{y}}^{d-2 r+1} f_{\mathbf{z}}^{d-2 r+1} \\
& -(\mathbf{y ~ w})(\mathbf{x ~ z})^{2 r-1} f_{\mathbf{y}}^{d-1} f_{\mathbf{w}}^{d-1} f_{\mathbf{x}}^{d-2 r+1} f_{\mathbf{z}}^{d-2 r+1} \\
& +(\mathbf{z ~ w})(\mathbf{x} \mathbf{y})^{2 r-1} f_{\mathbf{z}}^{d-1} f_{\mathbf{w}}^{d-1} f_{\mathbf{x}}^{d-2 r+1} f_{\mathbf{y}}^{d-2 r+1} \\
& -(\mathbf{z} \mathbf{y})(\mathbf{x} \mathbf{w})^{2 r-1} f_{\mathbf{z}}^{d-1} f_{\mathbf{y}}^{d-1} f_{\mathbf{x}}^{d-2 r+1} f_{\mathbf{w}}^{d-2 r+1}
\end{aligned}
$$

By construction, $\mathcal{F}$ is alternating in all four variables; hence $\zeta$ factors through $\wedge^{4} S_{d}$. The rationale behind this choice of $\zeta$ will be explained in Section 3.1.

2.6. The first calculation. Let us write (using the obvious notation)

$$
\mathcal{F}=\mathcal{T}(\mathbf{x y}, \mathbf{z w})-\mathcal{T}(\mathbf{x z}, \mathbf{y w})+\cdots-\mathcal{T}(\mathbf{z y}, \mathbf{x w}) .
$$

We should like to gauge the effect of the morphism $\beta_{3} \circ \beta_{2} \circ \beta_{1}$ on each summand in $\mathcal{F}$. The next two lemmata allow us to 'cancel' an $\Omega_{\mathbf{x y}}$ against an $(\mathbf{x} \mathbf{y})$.

LEMMA 2.3. Let $\mathcal{G}$ denote an arbitrary bi-homogeneous form of orders $p, q$ in $\mathbf{x}, \mathbf{y}$ respectively.

(a) For every $m \geq 1$,

$$
\Omega_{\mathbf{x y}}(\mathbf{x y})^{m} \mathcal{G}=m(p+q+m+1)(\mathbf{x} \mathbf{y})^{m-1} \mathcal{G}+(\mathbf{x} \mathbf{y})^{m} \Omega_{\mathbf{x y}} \mathcal{G}
$$

(b) For every $\ell \geq 1$,

$$
\Omega_{\mathbf{x} \mathbf{y}}^{\ell}(\mathbf{x} \mathbf{y}) \mathcal{G}=\ell(p+q-\ell+3) \Omega_{\mathbf{x y}}^{\ell-1} \mathcal{G}+(\mathbf{x} \mathbf{y}) \Omega_{\mathbf{x} \mathbf{y}}^{\ell} \mathcal{G}
$$


Proof. By straightforward differentiation,

$$
\begin{aligned}
\Omega_{\mathbf{x y}}(\mathbf{x} \mathbf{y}) \mathcal{G}= & 2 \mathcal{G}+\left(x_{1} \frac{\partial \mathcal{G}}{\partial x_{1}}+x_{2} \frac{\partial \mathcal{G}}{\partial x_{2}}\right)+\left(y_{1} \frac{\partial \mathcal{G}}{\partial y_{1}}+y_{2} \frac{\partial \mathcal{G}}{\partial y_{2}}\right) \\
& +\left(x_{1} y_{2}-x_{2} y_{1}\right)\left(\frac{\partial^{2} \mathcal{G}}{\partial x_{1} \partial y_{2}}-\frac{\partial^{2} \mathcal{G}}{\partial x_{2} \partial y_{1}}\right) \\
= & (p+q+2) \mathcal{G}+(\mathbf{x} \mathbf{y}) \Omega_{\mathbf{x y}} \mathcal{G} .
\end{aligned}
$$

Now part (a) follows by an easy induction on $m$ and (b) by one on $\ell$.

LEMMA 2.4. With $\mathcal{G}$ as above and $\ell, m \geq 0$,

$$
\left[\Omega_{\mathbf{x y}}^{\ell}(\mathbf{x} \mathbf{y})^{m} \mathcal{G}\right]_{\mathbf{x}, \mathbf{y} \rightarrow \mathbf{u}}= \begin{cases}\mu(p, q ; \ell, m)\left[\Omega_{\mathbf{x} \mathbf{y}}^{\ell-m} \mathcal{G}\right]_{\mathbf{x}, \mathbf{y} \rightarrow \mathbf{u}} & \text { if } \ell \geq m, \\ 0 & \text { otherwise }\end{cases}
$$

where

$$
\mu(p, q ; \ell, m)=\frac{\ell !}{(\ell-m) !} \frac{(p+q-\ell+2 m+1) !}{(p+q-\ell+m+1) !} .
$$

Proof. Using part (a) of the previous lemma for the connecting step, one shows by induction on $\ell$ that

$$
\Omega_{\mathbf{x y}}^{\ell}(\mathbf{x} \mathbf{y})^{m} \mathcal{G} \equiv \begin{cases}\mu(p, q ; \ell, m) \Omega_{\mathbf{x y}}^{\ell-m} \mathcal{G} & \text { if } 0 \leq \ell-m \leq \min (p, q), \\ 0 & \text { otherwise, }\end{cases}
$$

where $\equiv$ stands for congruence modulo $(\mathbf{x y})$. The result follows, because terms involving ( $\mathbf{x y}$ ) vanish after the substitution $\mathbf{x}, \mathbf{y} \rightarrow \mathbf{u}$.

As a consequence, the term $\mathcal{T}(\mathbf{z w}, \mathbf{x y})$ is annihilated by the operation $\Omega_{\mathbf{x y}}^{2 i-1}$ followed by $\{\mathbf{x}, \mathbf{y} \rightarrow \mathbf{u}\}$, unless $i=r$ (and hence necessarily $j=1$ ). In the latter case,

$$
\beta_{2} \circ \beta_{1}(\mathcal{T}(\mathbf{z w}, \mathbf{x y}))=\mathrm{h}(d, d, 2 r-1) \mathrm{h}(d, d, 1)\left[\Omega_{\mathbf{x y}}^{2 r-1} \Omega_{\mathbf{z w}} \circ \mathcal{T}(\mathbf{z w}, \mathbf{x y})\right]_{\{\mathbf{x}, \mathbf{y} \rightarrow \mathbf{u}\},\{\mathbf{z}, \mathbf{w} \rightarrow \mathbf{v}\}}
$$

evaluates to

$$
f_{\mathbf{u}}^{2 d-4 r+2} f_{\mathbf{v}}^{2 d-2}
$$

because

$$
\begin{aligned}
& \mathrm{h}(d, d, 2 r-1) \mathrm{h}(d, d, 1) \mu(d-2 r+1, d-2 r+1,2 r-1,2 r-1) \\
& \quad \times \mu(d-1, d-1,1,1)=1 .
\end{aligned}
$$

Then $\beta_{3}$ carries (11) into $f_{\mathbf{t}}^{4(d-r)}$. By the same argument, $\mathcal{T}(\mathbf{x y}, \mathbf{z w})$ goes to $f_{\mathbf{t}}^{4(d-r)}$ if $(i, j)=(1, r)$ and zero otherwise.

This disposes of two of the summands in $\mathcal{F}$; the rest of them will need more work. As an interlude, we will consider a preparatory example which illustrates the operation of $\Omega_{\mathbf{x y}}$ on a symbolic product involving $\mathbf{x}, \mathbf{y}$ (cf. [9, Section 3.2.5]). 
EXAMPLE 2.5. Let

$$
E=(\mathbf{x} \mathbf{z})^{7}(\mathbf{y} \mathbf{w})^{2} f_{\mathbf{x}} g_{\mathbf{x}}^{4} f_{\mathbf{y}}^{5}
$$

First we follow the calculation of $\Omega_{\mathbf{x y}} E$. The idea, in brief, is to pair an $\mathbf{x}$-factor with a $\mathbf{y}$-factor and contract them against each other. The following diagram shows all the types of $\mathbf{x}$ and $\mathbf{y}$ factors in $E$ and the possible pairings between them:

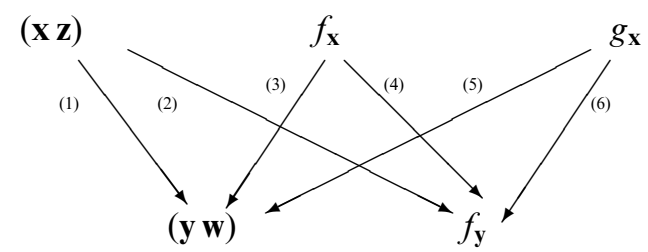

The equality $\Omega_{\mathbf{x y}}[(\mathbf{x} \mathbf{u})(\mathbf{y} \mathbf{v})]=(\mathbf{u} \mathbf{v})$ gives our basic rule: contracting (x u) against $(\mathbf{y} \mathbf{v})$ gives (uv). For instance, contraction along arrow (1) gives (zw). Introducing a phantom letter $\tilde{\mathbf{f}}=\left(-f_{2}, f_{1}\right)$, we can write $f_{\mathbf{x}}=(\mathbf{x} \tilde{\mathbf{f}})$, and hence contraction along arrow (3) gives $(\tilde{\mathbf{f}} \mathbf{w})=-f_{\mathbf{w}}$. Contraction along arrow (4) gives $(f f)=0$. Now $\Omega_{\mathbf{x y}} E$ is a sum of terms (quantified over all choices of contractions), where in each term the contracted factors are replaced by their result. Thus, $\Omega_{\mathbf{x y}} E=$

$$
\underbrace{14(\mathbf{z ~ w})(\mathbf{x ~ z})^{6}(\mathbf{y ~ w}) f_{\mathbf{x}} g_{\mathbf{x}}^{4} f_{\mathbf{y}}^{5}}_{\text {from arrow (1) }}+\underbrace{35 f_{\mathbf{z}}(\mathbf{x ~ z})^{6}(\mathbf{y ~ w})^{2} f_{\mathbf{x}} g_{\mathbf{x}}^{4} f_{\mathbf{y}}^{4}}_{\text {from arrow (2) }}+\cdots, \text { etc. }
$$

To calculate $\Omega_{\mathbf{x y}}^{2} E$ we must sum over all possible two-step sequences of contractions, taking account of available multiplicities. For instance, the sequences of arrows

$$
(1)(4), \quad(2)(2), \quad(3)(5)
$$

are allowed, but (3)(3) is not since there is only one $f_{\mathbf{x}}$ available. This gives $\Omega_{\mathbf{x y}}^{2} E=$

$$
\underbrace{840 f_{\mathbf{z}}^{2}(\mathbf{x ~ z})^{5}(\mathbf{y ~ w})^{2} f_{\mathbf{x}} g_{\mathbf{x}}^{4} f_{\mathbf{y}}^{3}}_{\text {from (2)(2) }}-\underbrace{120(g f) g_{\mathbf{w}}(\mathbf{x ~ z})^{7}(\mathbf{y ~ w}) f_{\mathbf{x}} g_{\mathbf{x}}^{2} f_{\mathbf{y}}^{4}}_{\text {from }(5)(6)}+\cdots, \text { etc. }
$$

If we treat the seven $(\mathbf{x} \mathbf{z})$ factors as notionally distinct, a sequence of two from them can be chosen in $7 ! / 5$ ! ways and similarly for $f_{\mathbf{y}}^{5}$. This gives the first coefficient as $\frac{7 !}{5 !} \frac{5 !}{3 !}=$ 840. Similarly, the second coefficient is $\frac{4 !}{2 !} \times 2 \times 5$. Notice that the sequence (6)(5) will give an additional term identical to the one coming from (5)(6).

2.7. We will now follow the evaluation of $\beta_{3} \circ \beta_{2} \circ \beta_{1} \circ \mathcal{T}(\mathbf{x w}, \mathbf{y z})$.

As a first step we have to remove $(2 i-1)$ factors each of type $\mathbf{x}, \mathbf{y}$ from $\mathcal{T}(\mathbf{x w}, \mathbf{y z})$. The available factors are respectively

$$
(\mathbf{x ~ w}) f_{\mathbf{x}}^{d-1} \text { and } \quad(\mathbf{y} \mathbf{z})^{2 r-1} f_{\mathbf{y}}^{d-2 r+1} .
$$


There are three choices:

(i) $(\mathbf{x ~ w}) f_{\mathbf{x}}^{2 i-2}$ and $(\mathbf{y} \mathbf{z})^{2 i-2} f_{\mathbf{y}}$;

(ii) $(\mathbf{x} \mathbf{w}) f_{\mathbf{x}}^{2 i-2}$ and $(\mathbf{y} \mathbf{z})^{2 i-1}$;

(iii) $\quad f_{\mathbf{x}}^{2 i-1}$ and $(\mathbf{y} \mathbf{z})^{2 i-1}$.

The possibilities are limited by the following constraint: since $f_{\mathbf{y}}$ can only be paired with $(\mathbf{x} \mathbf{w})$, no more than one copy of $f_{\mathbf{y}}$ can be chosen, and hence at least $2 i-2$ copies of $(\mathbf{y} \mathbf{z})$ must be chosen.

After contraction and the substitution $\{\mathbf{x}, \mathbf{y} \rightarrow \mathbf{u}\}$, choice (i) leads to the expression

$$
c_{I}(\mathbf{u ~ z})^{2 r-2 i+1} f_{\mathbf{u}}^{2 d-2 r-2 i+1} f_{\mathbf{z}}^{d-2 r+2 i-1} f_{\mathbf{w}}^{d}
$$

Here (and subsequently) $c_{I}, c_{I^{\prime}}$, etc. stand for some rational constants which will be determined later. Now we must remove $(2 j-1)$ factors each of type $\mathbf{z}, \mathbf{w}$ from (13). The choice is forced, namely

$$
\text { (i') }(\mathbf{u ~ z})^{2 j-1} \text { and } f_{\mathbf{w}}^{2 j-1} \text {. }
$$

After contraction and $\{\mathbf{z}, \mathbf{w} \rightarrow \mathbf{v}\}$, we get an expression

$$
-c_{I} c_{I^{\prime}}(\mathbf{u ~ v})^{2 r-2 i-2 j+2} f_{\mathbf{u}}^{2 d-2 r-2 i+2 j} f_{\mathbf{v}}^{2 d-2 r+2 i-2 j} .
$$

(The negative sign arises because contracting (u z) against $f_{\mathbf{w}}$ gives $-f_{\mathbf{u}}$.) Now $\beta_{3}$ will convert (14) into

$$
-c_{I} c_{I^{\prime}} f_{\mathbf{t}}^{4(d-r)}
$$

as a consequence of Lemma 2.4 .

2.8. Choice (ii) in (12) leads to the expression

$$
-c_{I I}(\mathbf{z ~ w}) \underbrace{(\mathbf{u ~ z})^{2 r-2 i} f_{\mathbf{u}}^{2 d-2 r-2 i+2} f_{\mathbf{w}}^{d-1} f_{\mathbf{z}}^{d-2 r+2 i-1}}_{\mathcal{G}},
$$

on which we have to operate on by $\Omega_{\mathbf{z w}}^{2 j-1}$. Using part (b) of Lemma 2.3,

$$
\Omega_{\mathbf{z w}}^{2 j-1}(\mathbf{z w}) \mathcal{G}=(2 j-1)(2 d-2 j+2) \Omega_{\mathbf{z w}}^{2 j-2} \mathcal{G}+(\mathbf{z w}) \Omega_{\mathbf{z w}}^{2 j-1} \mathcal{G} .
$$

After the substitution $\{\mathbf{z}, \mathbf{w} \rightarrow \mathbf{v}\}$, the second term goes away. In evaluating $\Omega_{\mathbf{z w}}^{2 j-2} \mathcal{G}$, we have a forced choice

$$
\text { (ii') }(\mathbf{u ~ z})^{2 j-2} \text { and } f_{\mathbf{w}}^{2 j-2} \text {, }
$$

leading to

$$
-(2 d-2 j+2)(2 j-1) c_{I I} c_{I I^{\prime}} f_{\mathbf{t}}^{4(d-r)}
$$


2.9. Choice (iii) (which is only possible if $2 i \leq d$ ), leads to

$$
-c_{I I I}(\mathbf{u w})(\mathbf{u z})^{2 r-2 i} f_{\mathbf{u}}^{2 d-2 r-2 i+1} f_{\mathbf{w}}^{d-1} f_{\mathbf{z}}^{d-2 r+2 i} .
$$

When applying $\Omega_{\mathbf{z w}}^{2 j-1}$, it further bifurcates into the two choices:

$$
\begin{aligned}
& \text { (iii') }(\mathbf{u ~ z})^{2 j-2} f_{\mathbf{z}} \text { and }(\mathbf{u ~ w}) f_{\mathbf{w}}^{2 j-2}, \\
& \text { (iii”) }(\mathbf{u ~ z})^{2 j-1} \text { and } f_{\mathbf{w}}^{2 j-1},
\end{aligned}
$$

which are dealt with similarly. In fact (iii') can arise only if

$$
2 j \leq d \quad \text { and } \quad r \geq i+j .
$$

Altogether we arrive at the expression

$$
\begin{aligned}
& \beta_{3} \circ \beta_{2} \circ \beta_{1} \circ \mathcal{T}(\mathbf{x w}, \mathbf{y z})=\mathrm{h}(d, d ; 2 i-1) \mathrm{h}(d, d ; 2 j-1) \\
& \quad \times\left(-c_{I} c_{I^{\prime}}-(2 d-2 j+2)(2 j-1) c_{I I} c_{I I^{\prime}}-c_{I I I} c_{I I I^{\prime}}+c_{I I I} c_{I I I^{\prime \prime}}\right) f_{\mathbf{t}}^{4(d-r)} .
\end{aligned}
$$

Using the recipe of Example 2.5, we get the constants

$$
\begin{aligned}
c_{I} & =(2 i-1)(d-2 r+1) \frac{(d-1) !}{(d-2 i+1) !} \frac{(2 r-1) !}{(2 r-2 i+1) !}, \\
c_{I^{\prime}} & =\frac{(2 r-2 i+1) ! d !}{(2 r-2 i-2 j+2) !(d-2 j+1) !}, \\
c_{I I} & =\frac{(2 i-1)(d-1) !(2 r-1) !}{(d-2 i+1) !(2 r-2 i) !} \\
c_{I I^{\prime}} & =\frac{(2 r-2 i) !(d-1) !}{(2 r-2 i-2 j+2) !(d-2 j+1) !}, \\
c_{I I I} & =\frac{(d-1) !(2 r-1) !}{(d-2 i) !(2 r-2 i) !} \\
c_{I I I^{\prime}} & =\frac{(2 j-1)(d-2 r+2 i)(2 r-2 i) !(d-1) !}{(2 r-2 i-2 j+2) !(d-2 j+1) !}, \\
c_{I I I^{\prime \prime}} & =\frac{(2 r-2 i) !(d-1) !}{(2 r-2 i-2 j+1) !(d-2 j) !} .
\end{aligned}
$$

If $2 i \leq d$ fails, then $c_{I I I}$ is zero by definition. Likewise, if the conditions in (17) are not satisfied, then $c_{I I I^{\prime \prime}}$ is understood to be zero. Recall that the prevailing hypotheses are

$$
3 \leq r \leq \frac{d+1}{2}, \quad 1 \leq i, j \leq r \quad \text { and } \quad i+j \leq r+1 .
$$

Therefore, any of extra conditions $2 i \leq d, 2 j \leq d$ and $i+j \leq r$ can only fail if respectively $d-2 i+1, d-2 j+1$ and $r-i-j+1$ vanish. Hence, the following expressions for $c_{I I I}$ and $c_{I I I^{\prime \prime}}$ hold unconditionally:

$$
\begin{aligned}
c_{I I I} & =\frac{(d-2 i+1)(d-1) !(2 r-1) !}{(d-2 i+1) !(2 r-2 i) !}, \\
c_{I I I^{\prime \prime}} & =\frac{(d-2 j+1)(2 r-2 i-2 j+2)(2 r-2 i) !(d-1) !}{(2 r-2 i-2 j+2) !(d-2 j+1) !} .
\end{aligned}
$$


Due to the symmetry in the situation, the rest of the terms

$$
-\mathcal{T}(\mathbf{x z}, \mathbf{y w}), \quad-\mathcal{T}(\mathbf{y w}, \mathbf{x z}), \quad-\mathcal{T}(\mathbf{z y}, \mathbf{x w})
$$

give identical evaluations. After some simplification, we arrive at the following formula:

2.10. Formula for $\theta_{i, j}$. Define $\delta_{i, j}$ to be 1 if $i=j$ and 0 otherwise. Let

$$
\begin{aligned}
\mathcal{N}_{1}= & \left(2 d i+2 d j-d r-2 i^{2}-2 j^{2}-2 d+3 i+3 j-2\right) \\
& \times d !(d-1) !(2 r-1) !(2 d-4 i+3) !(2 d-4 j+3) ! \\
\mathcal{N}_{2}= & (2 i-1) !(2 j-1) !(d-2 i+1) !(d-2 j+1) ! \\
& \times(2 d-2 i+2) !(2 d-2 j+2) !(2 r-2 i-2 j+2) !
\end{aligned}
$$

then

$$
\theta_{i, j}=\left(\delta_{i, 1} \delta_{j, r}+\delta_{i, r} \delta_{j, 1}-8 \frac{\mathcal{N}_{1}}{\mathcal{N}_{2}}\right)
$$

Evidently $\theta_{i, j}=\theta_{j, i}$. Therefore, in expression (9) one can combine the terms $(i, j)$ and $(j, i)$. Let $\epsilon_{i, j}=2$ if $i \neq j$ and 1 if $i=j$. Now let $\alpha_{i, j}=\epsilon_{i, j} \theta_{i, j}$. We have finally arrived at the required syzygy

$$
\mathcal{E}_{\zeta}: \sum_{(i, j)} \alpha_{i, j}\left(\mathcal{C}_{2 i-1}, \mathcal{C}_{2 j-1}\right)_{2(r-i-j+1)}=0
$$

where the sum is quantified over all pairs $(i, j)$ such that

$$
1 \leq i \leq j \leq r, \quad i+j \leq r+1 .
$$

The reader may check that for $d=7, r=3$, the syzygy becomes

$$
10\left(\mathcal{C}_{1}, \mathcal{C}_{1}\right)_{4}-\frac{80}{11}\left(\mathcal{C}_{1}, \mathcal{C}_{3}\right)_{2}-\frac{175}{121} \mathcal{C}_{3}^{2}+\frac{20}{21} \mathcal{C}_{1} \mathcal{C}_{5}=0
$$

which is the same as (4). We have (successfully) tested formula (20) in MAPLE on several examples.

2.11. Second calculation. In fact, formula (20) was first arrived at by a different path, namely by interpreting $\theta_{i, j}$ as (in essence) a 9-j symbol in the sense of the quantum theory of angular momentum (see [3, Section 7]).

We pick up the thread at the beginning of Section 2.5. The trajectory $f_{\mathbf{t}}^{4(d-r)} \longrightarrow$ $\mathcal{T}(\mathbf{x w}, \mathbf{y z})$ followed by $\beta_{3} \circ \beta_{2} \circ \beta_{1}$ is described by the sequence of morphisms

$$
\begin{aligned}
& S_{4(d-r)} \longrightarrow S_{2 d-2} \otimes S_{2 d-4 r+2} \longrightarrow\left(S_{d} \otimes S_{d}\right) \otimes\left(S_{d} \otimes S_{d}\right) \longrightarrow \\
& \left(S_{d} \otimes S_{d}\right) \otimes\left(S_{d} \otimes S_{d}\right) \longrightarrow S_{2 d-4 i+2} \otimes S_{2 d-4 j+2} \longrightarrow S_{4(d-r)} .
\end{aligned}
$$

Here the first two maps are natural injections; the last two are natural projections; and the one in the middle is the shuffling map,

$$
\left(v_{1} \otimes v_{2}\right) \otimes\left(v_{3} \otimes v_{4}\right) \longrightarrow\left(v_{1} \otimes v_{4}\right) \otimes\left(v_{2} \otimes v_{3}\right) .
$$


By Schur's lemma, the total composite must a multiple of the identity map $\operatorname{Id}_{S_{4(d-r)}}$. Up to an easily calculated factor (see [3, Section 7.9]), this multiple is the 9-j symbol,

$$
B=\left\{\begin{array}{ccc}
\frac{d}{2} & \frac{d}{2} & d-2 i+1 \\
\frac{d}{2} & \frac{d}{2} & d-2 j+1 \\
d-1 & d-2 r+1 & 2 d-2 r
\end{array}\right\} .
$$

Now interchange rows 1 and 2 of $B$; then interchange rows 1 and 3 of the new array; and finally interchange columns 2 and 3 . This gives an equivalent array,

$$
B^{\prime}=\left\{\begin{array}{ccc}
d-1 & 2 d-2 r & d-2 r+1 \\
\frac{d}{2} & d-2 i+1 & \frac{d}{2} \\
\frac{d}{2} & d-2 j+1 & \frac{d}{2}
\end{array}\right\} .
$$

Finally apply the Ališauskas-Jucys triple sum formula (see [3, Section 7.10]) to $B^{\prime}$. In the notation used there, the set $\Lambda$ of triples of indices which appear in the sum is contained in

$$
\{(d-2 r+1,2 j-1,0),(d-2 r+1,2 j-2,0),(d-2 r, 2 j-1,0)\},
$$

which reduces the sum to at most three easily manageable terms. The triple $(d-2 r+1$, $2 j-1,0)$ appears in the sum unless $i=r=\frac{d+1}{2}$. The triple $(d-2 r+1,2 j-2,0)$ always appears. Finally $(d-2 r, 2 j-1,0)$ appears unless $r=\frac{d+1}{2}$. One can remove the case discussion using the same trick which led to the unconditional formulae (18) and (19). After a little simplification, once again we get formula (20).

\section{Positivity.}

3.1. Operator norms. The next proposition will conclude the proof of Theorem 2.1.

Proposition 3.1. The coefficient $\alpha_{1, r}$ is non-zero and in fact strictly positive.

Proof. We will extensively use the material in [3, Section 7]. If $u: \mathcal{E}_{1} \longrightarrow \mathcal{E}_{2}$ denotes a linear map between Hilbert spaces, then $u^{*}: \mathcal{E}_{2} \longrightarrow \mathcal{E}_{1}$ denotes its adjoint. Recall that the Hilbert-Schmidt norm of $u$ is defined to be

$$
\|u\|_{\mathrm{HS}}=\sqrt{\operatorname{trace}\left(u^{*} \circ u\right)}
$$

For a composite $\mathcal{E}_{1} \stackrel{u}{\longrightarrow} \mathcal{E}_{2} \stackrel{v}{\longrightarrow} \mathcal{E}_{3}$, we have $(v \circ u)^{*}=u^{*} \circ v^{*}$.

In the notation of [3, Section 7], we write $\mathcal{H}_{\frac{m}{2}}$ for $S_{m}$, which carries a natural structure of a finite-dimensional Hilbert space. We will view $\zeta$ as a map from $\mathcal{H}_{2(d-r)}$ to $\left(\mathcal{H}_{\frac{d}{2}}\right)^{\otimes 4}$ via the natural inclusion $\wedge^{4} \mathcal{H}_{\frac{d}{2}} \hookrightarrow\left(\mathcal{H}_{\frac{d}{2}}\right)^{\otimes 4}$. Similarly, we view $\beta_{1}$ as originating 
from $\left(\mathcal{H}_{\frac{d}{2}}\right)^{\otimes 4}$ via the natural surjection,

$$
\begin{aligned}
\left(\mathcal{H}_{\frac{d}{2}}\right)^{\otimes 4} & \longrightarrow S_{2}\left(\wedge^{2} \mathcal{H}_{\frac{d}{2}}\right), \\
z_{1} \otimes z_{2} \otimes z_{3} \otimes z_{4} & \longrightarrow\left(z_{1} \wedge z_{2}\right) \cdot\left(z_{3} \wedge z_{4}\right) .
\end{aligned}
$$

Henceforth, throughout the proof, the symbol $\circlearrowleft$ will stand for some strictly positive constant which need not be specified. Recall that we have defined maps $\pi^{\mathrm{PHY}},{ }_{l}^{\mathrm{PHY}}$ such that

$$
\pi_{\frac{m}{2}, \frac{n}{2}, \frac{1}{2}(m+n-2 q)}^{\mathrm{PHY}}=\circlearrowleft \pi_{q}, \quad l_{\frac{m}{2}, \frac{n}{2}, \frac{1}{2}(m+n-2 q)}^{\mathrm{PHY}}=\circlearrowleft l_{q},
$$

in the notation of (1) and (2); moreover $\pi^{\mathrm{PHY}}=\left({ }_{l}^{\mathrm{PHY}}\right)^{*}$. We will show that

$$
\circlearrowleft \alpha_{1, r}=\|\zeta\|_{\mathrm{HS}}^{2} .
$$

First, observe that $\alpha_{1,1}=2(r-2)(2 r-1) \neq 0$; hence the map $\zeta$ is not identically zero (if the reader was not already so persuaded). If $\left(a_{s, t}\right)$ denotes the matrix representing $\zeta$ with respect to some orthonormal bases, then

$$
\operatorname{trace}\left(\zeta^{*} \circ \zeta\right)=\sum_{s, t}\left|a_{s, t}\right|^{2}>0
$$

hence it only remains to show (23) to complete the proof of the proposition.

Now specialise to $i=1, j=r$, and let $\psi=\beta_{3} \circ \beta_{2} \circ \beta_{1} \circ \zeta$. By definition, $\alpha_{1, r}=$ $2 \theta_{1, r}$, where $\psi=\theta_{1, r} \operatorname{Id}_{S_{4(d-r)}}$, and hence

$$
\theta_{1, r}=\frac{\operatorname{trace}(\psi)}{4(d-r)+1}
$$

Notice that, up to a positive multiplicative constant, the map $f_{\mathbf{t}}^{4(d-r)} \longrightarrow \mathcal{T}(\mathbf{x y}, \mathbf{z w})$ is the sequence

$$
\mathcal{H}_{2(d-r)} \longrightarrow \mathcal{H}_{d-1} \otimes \mathcal{H}_{d-2 r+1} \longrightarrow\left(\mathcal{H}_{\frac{d}{2}} \otimes \mathcal{H}_{\frac{d}{2}}\right) \otimes\left(\mathcal{H}_{\frac{d}{2}} \otimes \mathcal{H}_{\frac{d}{2}}\right)
$$

where the first map is $l_{j_{12} j_{3} J}^{\mathrm{PHY}}$, and the second is $l_{j_{1} j_{2} j_{12}}^{\mathrm{PHY}} \otimes l_{j_{3} j_{j} j_{3}}^{\mathrm{PHY}}$, with

$$
\begin{aligned}
& j_{12}=d-1, \quad j_{34}=d-2 r+1, \quad J=2(d-r) \quad \text { and } \\
& j_{1}=j_{2}=j_{3}=j_{4}=\frac{d}{2} .
\end{aligned}
$$

If we compose this with the alternation map

$$
\begin{aligned}
\mathcal{A}:\left(\mathcal{H}_{\frac{d}{2}}\right)^{\otimes 4} & \longrightarrow\left(\mathcal{H}_{\frac{d}{2}}\right)^{\otimes 4}, \\
z_{1} \otimes z_{2} \otimes z_{3} \otimes z_{4} & \longrightarrow \frac{1}{4 !} \sum_{\sigma \in \mathfrak{S}_{4}} \operatorname{sign}(\sigma) z_{\sigma(1)} \otimes z_{\sigma(2)} \otimes z_{\sigma(3)} \otimes z_{\sigma(4)},
\end{aligned}
$$

the net effect (up to a constant) is $\zeta: f_{\mathbf{t}}^{4(d-r)} \longrightarrow \mathcal{F}$. In other words,

$$
\zeta=\circlearrowleft \mathcal{A} \circ\left(l_{j_{1} j_{2} j_{12}}^{\mathrm{PHY}} \otimes l_{j_{3} j_{4} j_{34}}^{\mathrm{PHY}}\right) \circ l_{j_{12} j_{34} J}^{\mathrm{PHY}} .
$$


Now observe that $\beta_{3} \circ \beta_{2} \circ \beta_{1}$ is (up to a constant) the sequence of maps:

$$
\left(\mathcal{H}_{\frac{d}{2}} \otimes \mathcal{H}_{\frac{d}{2}}\right) \otimes\left(\mathcal{H}_{\frac{d}{2}} \otimes \mathcal{H}_{\frac{d}{2}}\right) \longrightarrow \mathcal{H}_{d-1} \otimes \mathcal{H}_{d-2 r+1} \longrightarrow \mathcal{H}_{2(d-r)},
$$

where the first map is $\pi_{j_{1} j_{2} j_{12}}^{\mathrm{PHY}} \otimes \pi_{j_{3} j_{4} j_{34}}^{\mathrm{PHY}}$, and the second is $\pi_{j_{12} j_{34} J}^{\mathrm{PHY}}$. Since the maps $\imath^{\mathrm{PHY}}$ and $\pi^{\mathrm{PHY}}$ (with identical subscripts) are mutually adjoint and $\mathcal{A}$ is a self-adjoint idempotent,

$$
\psi=\circlearrowleft \zeta^{*} \circ \zeta
$$

and the claim follows.

Indeed, it was this argument which led us to the correct guess for $\mathcal{F}$. One strategy to ensure that $\alpha_{1, r}$ does not vanish is to make it appear as the Hilbert-Schmidt norm of a non-zero operator. This prompted us to take the adjoint of $\beta_{3} \circ \beta_{2} \circ \beta_{1}$, which determines the first term in $\mathcal{F}$ and hence all the rest.

3.2. An elementary proof of Proposition 3.1. The result of Proposition 3.1 amounts to the inequality

$$
4\left(d r-2 r^{2}+3 r-1\right) \times \frac{(d-1) !(2 d-4 r+3) !}{(d-2 r+1) !(2 d-2 r+2) !}<1
$$

in the range $r \geq 3, d \geq 2 r-1$.

We include an elementary proof of this inequality. Let $\Gamma(r, d)$ denote the left-hand side of (24). First,

$$
\Gamma(r, 2 r-1)=\frac{2}{r}<1
$$

Let us write

$$
\frac{\Gamma(r, d+1)}{\Gamma(r, d)}=\frac{N}{D},
$$

where

$$
\begin{aligned}
& N=d\left(d r+4 r-2 r^{2}-1\right)(2 d-4 r+5), \\
& D=\left(d r-2 r^{2}+3 r-1\right)(2 d-2 r+3)(d-r+2) .
\end{aligned}
$$

Now observe that

$$
D-N=(r-1)(r-2)(2 r-1)(d-2 r+3)>0 ;
$$

hence $\Gamma(r, d+1)<\Gamma(r, d)$. This completes the proof.

Unfortunately this proof gives no insight into why the inequality should be true. It seems especially fortuitous that $D-N$ should admit such a tidy factorisation. For reasons already stated, we prefer the earlier argument.

The Hilbert-Schmidt idea also guided the construction of the closed form syzygy in [3, Section 2.14]; it can be used to provide an alternate proof of Lemma 2.3 therein. In [1] and [16, Proposition 5] one may find similar instances, where the non-vanishing of an algebraic expression produced by a tensorial construction is the key ingredient in a geometric result. 
3.3. $S L_{2}$-equivariant Plücker equations. There are many quadratic syzygies between the combinants $\mathcal{C}_{1}, \mathcal{C}_{3}, \ldots$, besides those given in Section 2.10; e.g. for $d=5$, we have an isomorphism

$$
\wedge^{4} S_{5} \simeq S_{8} \oplus S_{4} \oplus S_{0}
$$

hence there exists one such syzygy for each of the weights $6,8,10$. Using symbolic calculations (as in [11, Chapter 5]), they are seen to be

$$
\begin{aligned}
15\left(\mathcal{C}_{1}, \mathcal{C}_{1}\right)_{4}-\frac{60}{7}\left(\mathcal{C}_{1}, \mathcal{C}_{3}\right)_{2}-\frac{225}{98} \mathcal{C}_{3}^{2}+\mathcal{C}_{1} \mathcal{C}_{5} & =0 \\
6\left(\mathcal{C}_{1}, \mathcal{C}_{1}\right)_{6}+\frac{45}{7}\left(\mathcal{C}_{1}, \mathcal{C}_{3}\right)_{4}-\frac{150}{49}\left(\mathcal{C}_{3}, \mathcal{C}_{3}\right)_{2}-\mathcal{C}_{3} \mathcal{C}_{5} & =0 \\
\frac{15}{2}\left(\mathcal{C}_{1}, \mathcal{C}_{1}\right)_{8}+\frac{75}{14}\left(\mathcal{C}_{3}, \mathcal{C}_{3}\right)_{4}-\mathcal{C}_{5}^{2} & =0
\end{aligned}
$$

Only the first of these is given by formula (21). This leads to the following natural problem: for an arbitrary $d$, classify all the quadratic syzygies between the $\left\{\mathcal{C}_{2 i-1}\right\}$. This is tantamount to writing the Plücker equations for the Grassmannian $G\left(2, S_{d}\right)$ in $S L_{2}$-equivariant form.

Recall that we have a sequence of isomorphisms,

$$
\wedge^{4} S_{d} \simeq S_{4}\left(S_{d-3}\right) \simeq S_{d-3}\left(S_{4}\right)
$$

An explicit description of the first isomorphism is given in [2, Section 2.5]. The second is usually called Hermite reciprocity (see [17, Corollary 4.2.9]). It follows that quadratic syzygies between the $\left\{\mathcal{C}_{2 i-1}\right\}$ are in bijection with covariants of degree $d-3$ of a generic binary quartic.

4. A ternary example. Our main theorem leads to the analogous problem for $S L_{N}$-representations. To wit, let $V$ denote an $N$-dimensional vector space, and write $\mathbb{S}_{\lambda}$ for the Schur module $\mathbb{S}_{\lambda} V$ (see $[\mathbf{8}$, Lecture 6]). Assume that we are given a plethysm decomposition of Schur modules,

$$
\wedge^{2} \mathbb{S}_{\lambda} \simeq \bigoplus_{\nu}\left(\mathbb{S}_{v} \otimes \mathbb{k}^{M_{v}}\right)
$$

A general formula for $M_{v}$ in terms of Yamanouchi domino tableaux may be found in [4].

Let $\mathcal{C}=\left\{\mathcal{C}_{\nu}^{(i)}: \quad 1 \leq i \leq M_{\nu}\right\}$ denote the associated linear combinants of a pencil of tensors in $\mathbb{S}_{\lambda}$. It is a natural problem to find a sub-collection of $\mathcal{C}$ which determines the rest of them. We will now exhibit such an example in the ternary case. The symbolic formalism used below is explained in [3, Section 4].

4.1. A pencil of $(3,1)$-tensors. Assume $N=3$ and $\lambda=(3,1)$. We have a decomposition

$$
\wedge^{2} \mathbb{S}_{(3,1)} \simeq \underbrace{\mathbb{S}_{(5)} \oplus \mathbb{S}_{(5,3)} \oplus \mathbb{S}_{(4,1)} \oplus \mathbb{S}_{(3,2)} \oplus \mathbb{S}_{(1,1)}}_{\mathbf{E}}
$$


with projection morphisms $f_{\lambda}: \wedge^{2} \mathbb{S}_{(3,1)} \rightarrow \mathbb{S}_{\lambda}$. Let

$$
A=(a b \mathbf{u}) a_{\mathbf{x}}^{2}, \quad B=(c d \mathbf{u}) c_{\mathbf{x}}^{2}
$$

denote two 'generic' forms in $\mathbb{S}_{(3,1)}$, and write $\mathcal{C}_{\lambda}=f_{\lambda}(A \wedge B)$. Then we have symbolic formulae

$$
\begin{aligned}
\mathcal{C}_{(5)} & =(a b d) a_{\mathbf{x}}^{2} c_{\mathbf{x}}^{3}, \\
\mathcal{C}_{(5,3)} & =(a b \mathbf{u})(a c \mathbf{u})(a d \mathbf{u}) c_{\mathbf{x}}^{2}, \\
\mathcal{C}_{(4,1)} & =(a b d)(a c \mathbf{u}) a_{\mathbf{x}} c_{\mathbf{x}}^{2}-5(a b c)(a d \mathbf{u}) a_{\mathbf{x}} c_{\mathbf{x}}^{2}, \\
\mathcal{C}_{(3,2)} & =(a b d)(a c \mathbf{u})^{2} c_{\mathbf{x}}+(a b c)(a c \mathbf{u})(a d \mathbf{u}) c_{\mathbf{x}}, \\
\mathcal{C}_{(1,1)} & =(a b c)(a c d)(a c \mathbf{u}) .
\end{aligned}
$$

There is an exact sequence of $S L_{3}$-representations,

$$
0 \rightarrow \underbrace{\wedge^{4} \mathbb{S}_{(3,1)}}_{\mathcal{Q}} \rightarrow \mathbb{S}_{(2)}\left(\wedge^{2} \mathbb{S}_{(3,1)}\right) \rightarrow \mathbb{S}_{(2,2)}\left(\mathbb{S}_{(3,1)}\right) \rightarrow 0,
$$

and, as in the binary case, the irreducible sub-representations of $\mathcal{Q}$ correspond to the quadratic syzygies between the $\mathcal{C}_{\lambda}$.

Proposition 4.1. Either of the combinants $\mathcal{C}_{(3,2)}$ and $\mathcal{C}_{(1,1)}$ can be recovered from the set $\left\{\mathcal{C}_{(5)}, \mathcal{C}_{(5,3)}, \mathcal{C}_{(4,1)}\right\}$.

The result follows from an explicit calculation involving plethysms and projection maps. Taking our cue from the binary case, we look for sub-representations corresponding to $(5,0)+(3,2)=(8,2)$. Decomposing ${ }^{1} \mathcal{Q}$ and $\mathbb{S}_{2}(\mathbf{E})$ into irreducible summands, we found that they respectively contain 2 and 7 copies of $\mathbb{S}_{(8,2)}$. The latter come from tensor products of the summands in $\mathbf{E}$ taken two at a time; e.g. the morphism

$$
\mathbb{S}_{(5)} \otimes \mathbb{S}_{(5,3)} \longrightarrow \mathbb{S}_{(8,2)}
$$

is given by the formula

$$
a_{\mathbf{x}}^{5} \otimes(c d \mathbf{u})^{3} c_{\mathbf{x}}^{2} \rightarrow(a c d)(a d \mathbf{u})^{2} a_{\mathbf{x}}^{2} c_{\mathbf{x}}^{4}
$$

Let us write $\left\langle\mathcal{C}_{(5)}, \mathcal{C}_{(5,3)}\right\rangle$ for the image of $\mathcal{C}_{(5)} \otimes \mathcal{C}_{(5,3)}$ via this morphism. Once all the seven maps have been written down symbolically, it only remains to solve a system of linear equations to find the two-dimensional space of syzygies; this was done in MAPLE. One conveniently chosen syzygy is the following:

$$
\begin{aligned}
\mathcal{C}_{(5)} \mathcal{C}_{(3,2)}= & \frac{1}{7680}\left\langle\mathcal{C}_{(5)}, \mathcal{C}_{(5)}\right\rangle+\frac{1}{92160}\left\langle\mathcal{C}_{(5)}, \mathcal{C}_{(5,3)}\right\rangle-\frac{1}{5760}\left\langle\mathcal{C}_{(5)}, \mathcal{C}_{(4,1)}\right\rangle \\
& -\frac{1}{204800}\left\langle\mathcal{C}_{(5,3)}, \mathcal{C}_{(5,3)}\right\rangle-\frac{1}{51200}\left\langle\mathcal{C}_{(5,3)}, \mathcal{C}_{(4,1)}\right\rangle
\end{aligned}
$$

This gives a formula for $\mathcal{C}_{(3,2)}$ in terms of $\mathcal{C}_{(5)}, \mathcal{C}_{(5,3)}, \mathcal{C}_{(4,1)}$.

\footnotetext{
${ }^{1}$ The full decompositions are very lengthy, and it seems needless to list them here. All plethysm decomposition throughout this example were calculated using the 'SF' (symmetric functions) package for MAPLE written by John Stembridge.
} 
There are respectively 3 and 9 copies of $\mathbb{S}_{(6,1)}$ in $\mathcal{Q}$ and $\mathbb{S}_{2}(\mathbf{E})$, and the corresponding syzygies are found similarly. The syzygy

$$
\begin{aligned}
\mathcal{C}_{(5)} \mathcal{C}_{(1,1)}= & \frac{1}{25920}\left\langle\mathcal{C}_{(5)}, \mathcal{C}_{(5,3)}\right\rangle-\frac{1}{1728}\left\langle\mathcal{C}_{(5)}, \mathcal{C}_{(4,1)}\right\rangle-\frac{1}{864}\left\langle\mathcal{C}_{(5)}, \mathcal{C}_{(3,2)}\right\rangle \\
& -\frac{11}{69120}\left\langle\mathcal{C}_{(5,3)}, \mathcal{C}_{(4,1)}\right\rangle-\frac{5}{13824}\left\langle\mathcal{C}_{(5,3)}, \mathcal{C}_{(3,2)}\right\rangle-\frac{1}{4320}\left\langle\mathcal{C}_{(4,1)}, \mathcal{C}_{(3,2)}\right\rangle
\end{aligned}
$$

shows that $\mathcal{C}_{(1,1)}$ can be recovered from the rest of the combinants.

4.2. Symbolic descriptions. For the record, we state the symbolic expressions which were used to define the maps above. In formula (26), they are respectively

$$
\begin{aligned}
& \mathbb{S}_{(5)} \otimes \mathbb{S}_{(5)} \rightsquigarrow(a c \mathbf{u})^{2} a_{\mathbf{x}}^{3} c_{\mathbf{x}}^{3}, \\
& \mathbb{S}_{(5)} \otimes \mathbb{S}_{(5,3)} \rightsquigarrow(a c d)(a d \mathbf{u})^{2} a_{\mathbf{x}}^{2} c_{\mathbf{x}}^{4} \text {, } \\
& \mathbb{S}_{(5)} \otimes \mathbb{S}_{(4,1)} \rightsquigarrow(a c \mathbf{u})(a d \mathbf{u}) a_{\mathbf{x}}^{3} c_{\mathbf{x}}^{3}, \\
& \mathbb{S}_{(5,3)} \otimes \mathbb{S}_{(5,3)} \rightsquigarrow(a b d)^{2}(a b \mathbf{u})(a d \mathbf{u}) a_{\mathbf{x}} c_{\mathbf{x}}^{5} \text {, } \\
& \mathbb{S}_{(5,3)} \otimes \mathbb{S}_{(4,1)} \rightsquigarrow(a b d)(a b \mathbf{u})^{2} a_{\mathbf{x}}^{2} c_{\mathbf{x}}^{4} \text {, }
\end{aligned}
$$

where the target of each map is $\mathbb{S}_{(8,2)}$. In (27), they are respectively

$$
\begin{aligned}
& \mathbb{S}_{(5)} \otimes \mathbb{S}_{(5,3)} \rightsquigarrow(a c d)^{2}(a d \mathbf{u}) a_{\mathbf{x}}^{2} c_{\mathbf{x}}^{3}, \\
& \mathbb{S}_{(5)} \otimes \mathbb{S}_{(4,1)} \rightsquigarrow(a c d)(a c \mathbf{u}) a_{\mathbf{x}}^{3} c_{\mathbf{x}}^{2}, \\
& \mathbb{S}_{(5)} \otimes \mathbb{S}_{(3,2)} \rightsquigarrow(a c d)(a d \mathbf{u}) a_{\mathbf{x}}^{3} c_{\mathbf{x}}^{2}, \\
& \mathbb{S}_{(5,3)} \otimes \mathbb{S}_{(4,1)} \rightsquigarrow(a b c)(a b d)(a b \mathbf{u}) a_{\mathbf{x}}^{2} c_{\mathbf{x}}^{3}, \\
& \mathbb{S}_{(5,3)} \otimes \mathbb{S}_{(3,2)} \rightsquigarrow(a b d)^{2}(a b \mathbf{u}) a_{\mathbf{x}}^{2} c_{\mathbf{x}}^{3}, \\
& \mathbb{S}_{(4,1)} \otimes \mathbb{S}_{(3,2)} \rightsquigarrow(a b d)(a d \mathbf{u}) a_{\mathbf{x}}^{2} c_{\mathbf{x}}^{3},
\end{aligned}
$$

with target $\mathbb{S}_{(6,1)}$.

ACKNOWLEDGEMENTS. The second author was partly funded by a discovery grant from NSERC. Thanks are expressed to John Stembridge (author of the 'SF' package for Maple). Reference [4] was pointed out to us by Laurent Manivel. The Göttinger Digitalisierungszentrum, the University of Michigan Historical Library as well as Project Gutenberg have been useful in accessing some classical references, respectively $[10],[6,11]$ and $[9]$.

\section{REFERENCES}

1. A. Abdesselam and J. Chipalkatti, The bipartite Brill-Gordan locus and angular momentum, Transform. Grp. 11(3) (2006), 341-370.

2. A. Abdesselam and J. Chipalkatti, On the Wronskian combinants of binary forms, J. Pure Appl. Algebra 210(1) (2007), 43-61.

3. A. Abdesselam and J. Chipalkatti, The higher transvectants are redundant. Preprint arXiv:0801.1533v1 [math.AG] (2008).

4. C. Carré and B. Leclerc, Splitting the square of a Schur function into its symmetric and antisymmetric parts, J. Algebraic Combin. 4(3) (1995), 201-231. 
5. J. Chipalkatti, On the invariant theory of the Bézoutiant, Beiträge Algebra Geom. 47(2) (2006), 397-417. 1872).

6. A. Clebsch, Theorie der Binaren Algebraischen Formen (Teubner, Leipzig, Germany,

7. I. Dolgachev, Lectures on invariant theory, London Mathematical Society Lecture Notes No. 296 (Cambridge University Press, Cambridge, 2003).

8. W. Fulton and J. Harris, Representation theory: A first course, Graduate Texts in Mathematics. (Springer, New York, 1991).

9. O. Glenn, The theory of invariants (Ginn and Co., Boston, 1915).

10. P. Gordan, Ueber Combinanten, Math. Ann. 5 (1872), 95-122.

11. J. H. Grace and A. Young, The algebra of invariants (Chelsea, New York, 1962).

12. M. Meulien, Sur les invariants des pinceaux de formes quintiques binaires, Ann. Inst. Fourier (Grenoble) 54 (2004), 21-51.

13. P. E. Newstead, Covariants of pencils of binary cubics, Proc. R. Soc. Edinburgh Sect. A, 91(3-4) (1981-1982), 181-183.

14. P. Olver, Classical invariant theory, London Mathematical Society Student Texts (Cambridge University Press, Cambridge, 1999).

15. W. Shenton, Linear combinants of systems of binary forms, with the syzygies of the second degree connecting them, Am. J. Math. 37(3) (1915), 247-271.

16. N. I. Shepherd-Barron, The rationality of some moduli spaces of plane curves, Compositio Math. 67 (1988), 51-88.

17. B. Sturmfels, Algorithms in invariant theory, Texts and Monographs in Symbolic Computation (Springer, Vienna, 1993).

18. C. T. C. Wall, Pencils of binary quartics, Rend. Sem. Mat. Univ. Padova 99 (1998), $197-217$. 